\title{
A Semi-automated Method for the Measurement of the Fetal Nuchal Translucency in Ultrasound Images
}

\author{
Ezio Catanzariti ${ }^{1}$, Giovanni Fusco ${ }^{1}$, Francesco Isgrò ${ }^{1}$, Salvatore Masecchia ${ }^{1}$, \\ Roberto Prevete $^{2}$, and Matteo Santoro ${ }^{2}$ \\ 1 Dipartimento di Scienze Fisiche \\ Università degli Studi di Napoli Federico II \\ Napoli, Italy \\ 2 Dipartimento di Informatica e Scienze dell'Informazione \\ Università degli Studi di Genova \\ Genova, Italy
}

\begin{abstract}
Nowadays the measurement of the nuchal translucency thickness is being used as part of routine ultrasound scanning during the end of the first trimester of pregnancy, for the screening of chromosomal defects, as trisomy 21. Currently, the measurement is being performed manually by physicians. The measurement can take a long time for being accomplished, needs to be performed by highly skilled operators, and is prone to errors. In this paper we present an algorithm that automatically detects the border of the nuchal translucency, once a region of interest has been manually identified. The algorithm is based on the minimisation of a cost function, and the optimisation is performed using the dynamic programming paradigm. The method we present overcomes several of the drawbacks present in the state of the art algorithms.
\end{abstract}

\section{Introduction}

It is getting more and more common for women to carry the pregnancy when over thirty. Since the risk of a foetus with chromosomal defects increases with the age of the mother [1, it is now very important to have screening techniques for detecting foetuses with chromosomal defects during early pregnancy.

There are two categories of screening procedures: invasive and non-invasive. Invasive diagnosis methods, as amniocentesis and chorionic villus sampling, do give a definitive answer, but need to be performed by trained and experienced operators, and studies have shown that they increase of about 1-2\% the risk of fetal loss 2]. During the last thirty years research has aimed at developing non-invasive methods for prenatal diagnosis of fetal cells or cell-free DNA found in the maternal blood [2. However the outcome is that the analysis of maternal blood is more likely to be used as a method for assessment of risk, rather than as a non-invasive prenatal diagnosis of chromosomal defects. Moreover different studies have brought contradictory evidence concerning the concentration of cellfree fetal DNA in trisomy 21 foetuses: some studies reported that the levels are

P. Foggia, C. Sansone, and M. Vento (Eds.): ICIAP 2009, LNCS 5716, pp. 613-622, 2009.

(C) Springer-Verlag Berlin Heidelberg 2009 
increased and others reported that there is no difference from chromosomally normal pregnancies [2].

Despite the fact that no definitive non-invasive diagnosis is available at the moment, it is still possible to calculate a risk for chromosomal defects considering both patient-specific information and foetus related data. Among the former maternal age and the presence of previous affected pregnancies are the most significative. For the latter indicative data are the thickness of nuchal translucency (the sonographic appearance of sub-cutaneous accumulation of fluid behind the fetal neck in the first trimester of pregnancy) and/or the absence of the nose [3,2]. In fact it has been shown that about $75 \%$ of trisomy 21 foetuses have increased nuchal translucency thickness and 60-70\% have absent nasal bone 2. Studies in the mid 90s have shown that in normal pregnancies fetal nuchal translucency thickness increases with gestation during the first trimester [4], and that in trisomy 21 and other major chromosomal defects fetal nuchal translucency thickness is increased more than in normal pregnancies, and that the risk for trisomies can be derived by multiplying the a priori maternal age and gestational-related risk by a likelihood ratio, which depends on the degree of deviation in fetal nuchal translucency measurement from the normal median for that crown-rump length [2. It was estimated that, in a pregnant population with a mean maternal age of 28 years, using the risk cut-off of 1 in 300 to define the screen positive group would detect about $80 \%$ of trisomy 21 foetuses for a false positive rate of $5 \%$. Therefore this test, together with other indicators, is used to check if the foetus can be at risk, and in the case of a positive answer it is suggested to take a chorionic villus sampling or amniocentesis for a definitive answer. These non-invasive diagnosis methods are used only to avoid amniocentesis when it is not necessary, that is when the risk of a trisomy 21 foetus is low enough.

Nuchal translucency (henceforth NT) is the subcutaneous fluid-filled space between the back of the neck of a foetus and the overlying skin [2]. In figure 1 it is shown an ultrasound image of the foetus with the NT highlighted, and the thickness measurement superimposed. The NT thickness is defined as the maximum thickness of the translucent space, that is the dark area between the two echogenic lines, the white borders delimiting the NT (see figure1). Currently manual measurement is being performed by physicians using electronic callipers placed on the two echogenic lines on the screen of the sonograph [6] (see figure 1). The measurement takes an average of 15-20 minute for being accomplished, but it can take up to 35 minutes. There are two main reasons for this long amount of time. The first one is that that the NT thickness is measured in the saggital section of the foetus, and therefore the operator must look to find the correct position for the sensor and determine the best image for taking the measurement. The second one is that the measurement itself can be very tedious as the task of accurately placing callipers is very difficult. The measurement, that for its difficulty needs to be performed by highly skilled operators [6], is therefore prone to errors, and intra-observer and inter-observer repeatability can be questioned [7. 

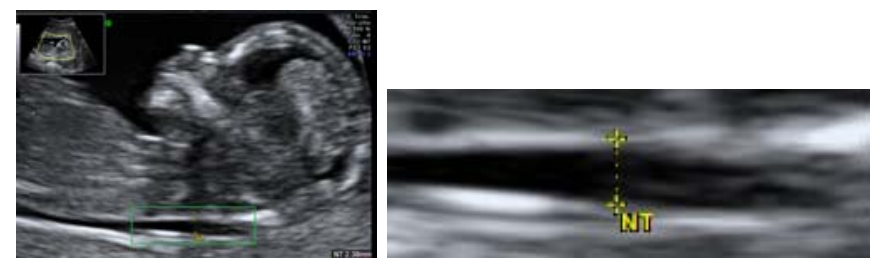

Fig. 1. Example of ultrasound image of the foetus. Left an image with the ROI containing the NT highlighted. Right the ROI with the result of manual measurement.

The research work presented in this paper is part of a work aiming to design and develop appropriate software tools capable to make the whole process of non-invasive screening easier for the operator, and, at the same time, more robust removing the issue of intra-observer and inter-observer repeatability. The advantage of an automated system is twofold. First, the measurement would become objective and repeatable, making the whole test more reliable, with the possible side effect of reducing the number of invasive screening. Second, with an automatic measurement the time necessary for a single diagnosis would be shortened, cutting down the amount of time patients have to wait before being examined, and at the same time, increasing the number of screenings that can be performed daily.

In this paper we present an algorithm for the automatic identification of the two echogenic lines and measurement of the NT thickness, once the human operator has identified a region of interest in the image. The algorithm presented starts from the work reported in [8] and overcomes some of the drawbacks present in the current literature. In particular the algorithm for the identification of the echogenic lines is an original piece of work, as it is based on the optimisation of a completely different cost function. The main advantages of our algorithm with respect to the previous work is that it is general, as it does not depend on weights that must be tuned for each image, and that always returns continuous borders.

The paper is organised as follows. The next Section reviews the related work. Section 3 describes the algorithm proposed in this paper, and Section 4 outlines the procedure for the automatic measurement of the NT thickness. Results are shown and discussed in Section 5. Section 6 is left to the final remarks.

\section{Previous Work}

Although the literature on medical image analysis is huge and covers a wide spectrum of topics (e.g., analysis of MRI images, mammograms) not so much work has been dedicated to automatic fetal measurements from ultrasound images; in fact the first few papers started appearing about 10 years ago [9]. The lack of too many scientific papers is due to the fact that ultrasound fetal images are very difficult data to deal with. The fetal ultrasound measurement considered 
in literature are typically: bi-parietal diameter, head circumference, abdominal circumference and femur length [9,10,11,12, and the problem is still far from being solved, in particular because of the complexity of the object appearance, high presence of noise and shadows, and because of the amount of information that need to be processed.

Among the work done on automatic fetal measurements the topics of automatic measurement of the nuchal translucency thickness and of automatic detection of the nose bone have not been addressed by many authors. Actually we tracked down only two papers on the measurement of the nuchal translucency [13,8, and none at all on the detection of the nose bone. The two papers mentioned, as the present one, differ in the method for determining the NT borders

The system in 13 helps the user determining the borders of the NT enhancing the edges with simple Sobel operator and using a threshold specified by the user on the magnitude of the gradient for detecting a variable number of image edges. The borders are identified manually selecting two point, one on each borders, and entirely determined via a flood-fill operation. The NT thickness is then automatically measured using the same algorithm adopted in 8 and in this work, and that will be outlined in section 4

Less intervention of the operator is required for the method given in [ $[$, where the image is first preprocessed with a CED filter 14 for reducing the speckle noise typical of ultrasound images. The NT borders detection on a ROI manually selected by an operator is addressed as a global optimisation problem based on the dynamic programming (DP) paradigm. The cost function adopted is

$$
\sum_{j=1}^{k} w_{1} f_{1}\left(p_{j}\right)+w_{2} f_{2}\left(p_{j}\right)+w_{3} f_{3}\left(p_{j}\right)+w_{4} g\left(p_{j-1}, p_{j}\right)
$$

where the $w_{i}$ are weights. The terms $f_{1}(p)$ and $f_{2}(p)$ are respectively the average value of an interval of pixels above and below the pixel $p$, and are introduced to ensure that $p$ is a transition point between a dark flat area and a bright flat area; $f_{3}(p)$ is the vertical component of the image gradient; the term $g\left(p_{j-1}, p_{j}\right)$ is a term introduced for ensuring border continuity, and is the distance of the pixel $p$ from a reference line, which is chosen as a straight line when computing the lower border, and the lower border itself when computing the upper border. The weights are not necessarily the same for both borders. The choice of the cost function appears based on empiric considerations, and the quality of results is strongly dependent on the weights. In fact, our implementation of this method
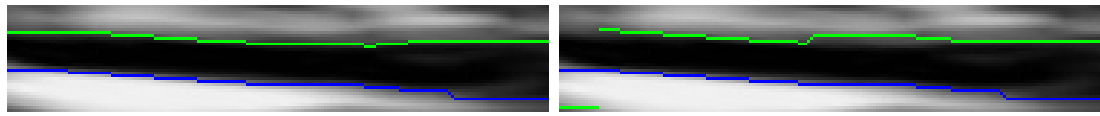

Fig. 2. Results from the algorithm in [8] with different weights. A not careful choice of the weights can produce discontinuous borders. 
showed that a bad choice of the weights can lead to discontinuous borders as shown in figure 2 .

\section{$3 \quad$ NT Borders Estimation}

The method for estimating the echogenic lines that we describe in this section overcomes some of the drawbacks of the algorithm presented in [8] and briefly reviewed in the previous section. Our method, summarised in table 1, is still semi-automatic, as it needs that the image region containing the NT is manually identified. Our algorithm differs from the one in [8] in several aspects. First, the cost function that we propose does not depend on any weight that need to be carefully tuned on each image, making the algorithm suitable for an automated system. Second, we introduce explicitly a term in the cost function that enforces the continuity of the border. Third, we substitute the terms $f_{1}$ and $f_{2}$ in equation 1 with a function of the zero-crossing direction that penalises edge points where the change between dark and bright pixels is not in the right direction. A last difference between the two algorithms is that we completely eliminated the $g$ term when computing the lower border, and substituted it with a term that constrains the upper border to remain above the lower border.

The method we propose for estimating the borders of the NT in the selected ROI is as follows. Considering a ROI of dimensions NxM, borders are considered as polylines:

$$
B_{N}=\left\{p_{1}, p_{2}, \cdots, p_{N-1}, p_{N}\right\}
$$

where $p_{j-1}$ and $p_{j}$ are adjacent pixels and $\mathrm{N}$ is the total length of the border. The cost function we introduce for the detection of the upper and lower border are different from each other in signs and in number of terms. For the lower border, that is computed first, the cost function to minimise is:

$$
\sum_{j=1}^{N}-\frac{\partial f}{\partial y}\left(p_{j}\right)+Z_{l}\left(p_{j}\right)+f_{a d j}\left(p_{j}\right.
$$

while for the detection of the upper border the cost function is:

$$
\sum_{j=1}^{N} \frac{\partial f}{\partial y}\left(p_{j}\right)+Z_{u}\left(p_{j}\right)+f_{a d j}\left(p_{j}, p_{j-1}\right)+f_{p o s}\left(B_{j}^{L}, p_{j}\right) .
$$

The first term, common to both cost functions, consists of the image derivative along the vertical direction, in order to consider the energy deriving from the image features as edges or lines.

Second order derivatives are computed along the gradient direction $\theta_{p}$, so that, by detecting zero-crossings, we can get more precise information about edges location, and taking account of their direction we can decide if they represent a transition from high intensity to darker region (upper border) or vice-versa 
Table 1. Summary of the algorithm

1. Compute image gradient $\nabla f$

2. For each point of $p$, compute gradient direction $\theta_{p}$

3. Compute second order derivative $\frac{\partial^{2} f}{\partial \theta_{p}^{2}}$ along gradient direction

4. Compute zero-crossing functions $Z_{u}$ and $Z_{l}$ as follows:

(a) $Z_{u}(x, y)=\left\{\begin{array}{l}0 \text { if } \frac{\partial^{2} f}{\partial \theta_{p}^{2}}(p) \times \frac{\partial^{2} f}{\partial \theta_{p}^{2}}(q)<0 \\ \zeta \text { otherwise }\end{array}\right.$ where $q$ is the point preceding $p$ along $\theta_{p}$

(b) $Z_{l}(x, y)=\left\{\begin{array}{l}0 \frac{\partial^{2} f}{\partial \theta_{P}^{2}}(p) \times \frac{\partial^{2} f}{\partial \theta_{p}^{2}}(t)<0 \\ \zeta \text { otherwise }\end{array}\right.$

where $t$ is the point following along $y$ and $\zeta>0$.

5. Considering a border $\mathrm{B}$ as a polyline, $B=\left\{p_{1}, p_{2}, \cdots, p_{N}\right\}$, detect lower border using Dynamic Programming (DP):

$C_{l}\left(B_{1}^{L}\right)=-\frac{\partial f}{\partial y}\left(p_{1}\right)+Z_{l}(x, y)$

$C_{l}\left(B_{n}^{L}\right)=C_{l}\left(B_{n-1}\right)+\left\{-\frac{\partial f}{\partial y}\left(p_{n}\right)+Z_{l}\left(p_{n}\right)+f_{a d j}\left(p_{n}, p_{n-1}\right)\right\}$

detect upper border using DP:

$C_{u}\left(B_{1}^{U}\right)=\frac{\partial f}{\partial y}\left(p_{1}\right)+Z_{u}(x, y)+f_{\text {pos }}\left(B_{1}^{L}, p_{1}\right)$

$C_{u}\left(B_{n}^{U}\right)=C_{l}\left(B_{n-1}+\left\{\frac{\partial f}{\partial y}\left(p_{n}\right)+Z_{u}\left(p_{n}\right)+f_{a d j}\left(p_{n}, p_{n-1}\right)+f_{\text {pos }}\left(B_{n}^{L}, p_{n}\right)\right\}\right.$

where

$f_{a d j}\left(p_{n}, p_{n-1}\right)=\left\{\begin{array}{l}0 \text { if } d\left(p_{n}, p_{n-1}\right) \leq 1, \\ \zeta \text { otherwise }\end{array}\right.$ and $f_{\text {pos }}$ is a sigmoid function of the vertical distance between a candidate point on the upper border and its corresponding on the lower border.

(lower border). The second term of the cost function consists of the non zerocrossings penalties in order that image points that are not on edges will have a high cost. These terms can be expressed for the upper border as

$$
Z_{u}(p)=\left\{\begin{array}{l}
0 \text { if } \frac{\partial^{2} f}{\partial \theta_{p}^{2}}(p) \times \frac{\partial^{2} f}{\partial \theta_{p}^{2}}(q)<0 \\
\zeta \text { otherwise },
\end{array}\right.
$$

and for the lower border as

$$
Z_{l}(p)=\left\{\begin{array}{l}
0 \frac{\partial^{2} f}{\partial \theta_{p}^{2}}(p) \times \frac{\partial^{2} f}{\partial \theta_{p}^{2}}(t)<0 \\
\zeta \text { otherwise }
\end{array}\right.
$$

where $q$ is the pixel preceding $p$ along the gradient direction, and $t$ is the pixel next to $p$ along the gradient direction, and $\zeta>0$.

In order to enforce the continuity of the borders we introduce a third term, $f_{a d j}$, in the cost function, that penalises a polyline where consecutive pixels have distance larger than one pixel:

$$
f_{a d j}\left(p_{j}, p_{j-1}\right)=\left\{\begin{array}{l}
0 \text { if } d\left(p_{j}, p_{j-1}\right) \leq 1, \\
\zeta \text { otherwise }
\end{array}\right.
$$

We also introduced a fourth term, $f_{\text {pos }}$, exclusively in the cost function used for the detection of the upper border. This term consists of a sigmoidal function 
which weighs the relative distance between candidate points of the upper border and the corresponding points on the lower border. In this way we strongly constrain the upper border to be made up of points that stay above the lower border.

The problem of the border detection is then solved optimising the cost functions $C_{l}$ and $C_{u}$ defined above. The solution has to be found globally in the ROI in order to avoid local minima that can be caused, for example, by residual speckle noise in the image.

\section{NT Thickness Measurement}

The last step is to estimate the maximum distance between the two borders we find. We achieve this using the same technique described in [13, and used in 8 . Let $B_{l}$ and $B_{u}$ be the lower and upper border respectively. The algorithm, depicted in figure 3 proceeds as follows.

1. Compute the median points $m_{j}$ between $p_{j} \epsilon B_{l}$ and $q_{j} \epsilon B_{u}$;

2. Compute the line $L_{j_{U}}$ using the points $p_{i} \epsilon B_{l}, i=j-2, \cdots, j+2$;

3. Compute the line $L_{j_{L}}$ using the points $q_{i} \epsilon B_{u}, i=j-2, \cdots, j+2$;

4. Compute the line $M_{j}$ using the computed median points $m_{i}, i=j-2, \cdots, j+2$;

5. Compute the line $O_{j}$ orthogonal to $M_{j}$ in $m_{j}$;

6. Compute the intersection points $r_{j 1}$ and $r_{j 2}$ between $O_{j}$ and the two lines $L_{j U}$ and $L_{j L}$, and store the distance $d_{j}=\left\|r_{j 1}-r_{j 2}\right\| ;$

7. Take the NT thickness as $\max _{j}\left(d_{j}\right)$; the relative points $r_{j 1}$ and $r_{j 2}$ are taken as the artificial position for the callipers.

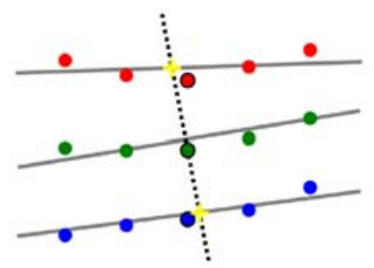

Fig. 3. Estimation of the NT thickness

\section{Results}

We run the algorithm on a set of ultrasound images, where the region of interests containing the NT have been identified by an expert physician. The images have been acquired using a GE Voluson E8, that automatically applies some digital filtering to the images for removing the noise. Unfortunately it was not possible to know what filtering is performed as the whole product is protected by a patent. After a few trials we decided not to use any kind of filtering, not even a 


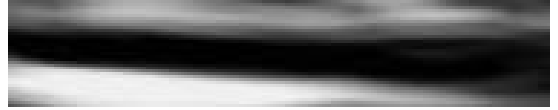

(a)

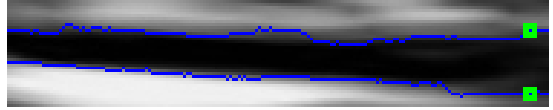

(b)

Fig. 4. Original image and result of our algorithm. The result of the algorithm in 8 is in figure 2 .
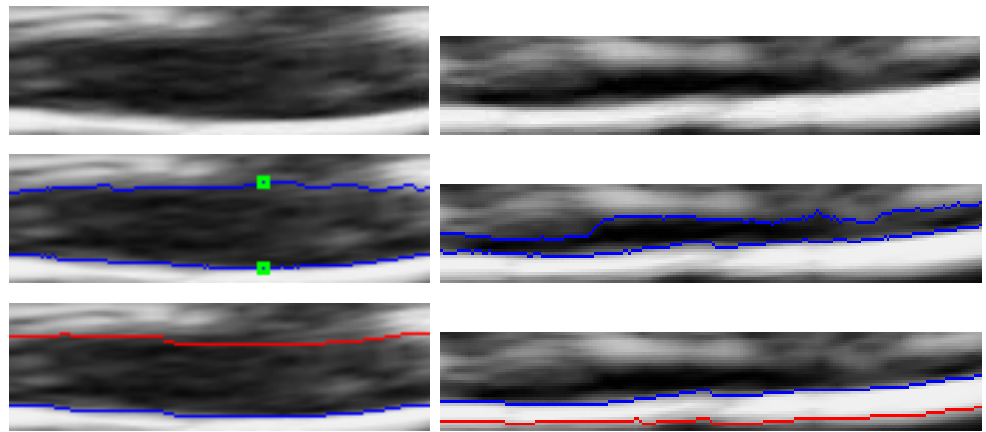

Fig. 5. Results of both algorithms for determining the NT borders. Top row original images. Middle row output of our algorithm. Bottom row result from the algorithm in 8 .

smoothing filter, before calculating the gradient, relying entirely on the filtering operated by the system.

The data produced by the acquisition device are also in a proprietary format, so that we were not able to recover the real image resolution, necessary for testing the NT thickness recovered against the measure obtained manually by the physician. Moreover all the images appear to have different resolutions. Because of this technical difficulty we were able only to test our algorithm against the algorithm presented in [8, for the quality of the echogenic lines recovered. It is worth to point out that no quantitative analysis of the performance of the automatic measurement of the NT thickness has been published so far.

In figures 4 and 5 the results of our algorithm, with markers for the points $r_{j 1}$ and $r_{j 2}$, are shown. In figure $4(\mathrm{a})$ is the original image relative to the results of our implementation of the algorithm in [8] shown in 2, and in figure 4(b) is the result of our algorithm. It can be noted how the border in figure 2 tends to be piecewise rectilinear, while the one produced by our algorithm follows the contour of the NT more closely. This difference in the performance is due to the $g$ term in the cost function 1, that forces the lower border to be rectilinear, and therefore extends this behaviour to the upper border. The contour returned by our algorithm is not particularly smooth, and we are planning to introduce a smoothness term in the cost function.

A similar behaviour is shown in the examples displayed in figure 5, where on the top row are the original images, our result is in the middle row, and the 

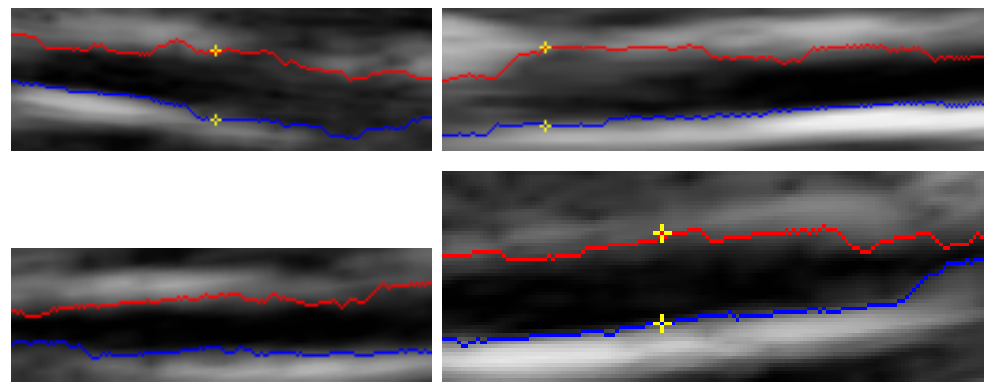

Fig. 6. Results of our algorithm for determining the NT borders

results from the algorithm in [8] is shown in the bottom row. It must be noted how the best result we managed to obtain with the competitor algorithm for the second image completely missed the NT.

In figure [ a few more echogenic lines determined by our algorithm are shown.

\section{Conclusions}

In this paper we presented an algorithm for the automatic estimation of the borders of the nuchal translucency from ultrasound images of the foetus, that is a key step towards measurement of the NT thickness. The NT measure is obtained using a state of the art method. The main assumption we make is that the region containing the NT is manually identified.

This work is a first step of a larger work the aim of which is the realisation of a fully automated system for the trisomy 21 screening, that will include automatic identification of the NT, the NT thickness measurement, and the test for the presence of the nose bone.

Next steps in the pipeline will be an extensive validation of the automatic measurement method, that at the best of our knowledge, is missing in the literature, and the development of the automatic identification of the NT.

\section{Acknowledgements}

The authors wish to thank dr. Rosalia Musone and dr. Enrico Colosi for providing the images and for their kind technical help.

\section{References}

1. Hecht, C., Hook, E.: The imprecision in rates of down syndrome by 1-year maternal age intervals: a critical analysis of rates used in biochemical screening. Prenatal Diagnosis 14, 729-738 (1994)

2. Nicolaides, K., Sebire, N., Snijders, R.: The 11-14 weeks scan: the diagnosis of fetal abnormalities. Parthenon Publishing, New York (1999) 
3. Nicolaides, K., Azar, G., Byrne, D., Mansur, C., Marrks, K.: Fetal nuchal translucency: ultrasound screening for chromosomal defects in first trimester of pregnancy. BMJ 304, 867-869 (1992)

4. Pandya, P., Snijders, R., Johnson, S., Brizot, M., Nicolaides, K.: Screening for fetal trisomies by maternal age and fetal nuchal translucency thickness at 10 to 14 weeks of gestation. BJOG 102, 957-962 (1995)

5. Snijders, R., Noble, P., Souka, A., Nicolaides, K.: UK multicentre project on assesment of risk of trimomy 21 by maternal age and fetal nuchal translucency thickness at 10-14 weeks of gestation. Lancet 352, 343-346 (1998)

6. Abuhamad, A.: Technical aspects of nuchal translucency measurement. Seminars in perinatology 29, 376-379 (2006)

7. Pandya, P., Altman, D., Brizot, M., Pettersen, H., Nicolaides, K.: Repeatability of measurement of fetal nuchal translucency thickness. Ultrasound in Obstetrics and Gynecology 5, 334-337 (1995)

8. Lee, Y., Kim, M., Kim, M.: Robust border enhancement and detection for measurement of fetal nuchal translucency in ultrasound images. Medical and Biological Engineering and Computing 45(11), 1143-1152 (2007)

9. Chalana, V., Winter, T., Cyr, D., Haynor, D., Kim, Y.: Automatic fetal head measurements from sonographic images. Academic Radiology 3(8), 628-635 (1996)

10. Hanna, C., Youssef, M.: Automated measurements in obstetric ultrasound images. In: Proceedings of the International Conference Image Processing, vol. 3, pp. $714-717$ (1997)

11. Carneiro, G., Georgescu, B., Good, S., Comaniciu, D.: Detection and measurement of fetal anatomies from ultrasound images using a constrained probabilistic boosting tree. IEEE Transactions on Medical Imaging 27(9), 1342-1355 (2008)

12. Pathak, S., Chalana, V., Kim, Y.: Interactive automatic fetal head measurements from ultrasound images using multimedia computer technology. Ultrasound in Medicine and Biology 23(5), 665-673 (1997)

13. Bernardino, F., Cardoso, R., Montenegro, N., Bernardes, J., de Sa, J.M.: Semiautomated ultrasonographic measurement of fetal nuchal translucency using a computer software tool. Ultrasound in Medicine and Biology 24(1), 51-54 (1998)

14. Weikert, J.: Coherence-enhancing diffusion filtering. International Journal of Computer Vision 31(2-3), 111-127 (1999) 